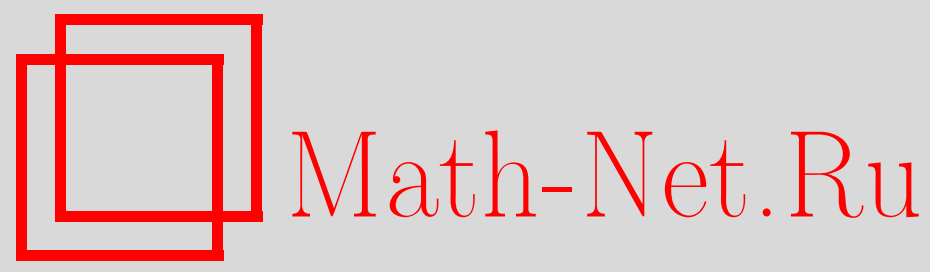

В. Н. Безверхний, И. В. Добрынина, О нормализаторах некоторых классов подгрупп в группах кос, Матем. заметкu, 2003, том 74, выпуск 1, 19-31

DOI: https://doi.org/10.4213/mzm242

Использование Общероссийского математического портала Math-Net.Ru подразумевает, что вы прочитали и согласны с пользовательским соглашением http://www.mathnet.ru/rus/agreement

Параметры загрузки:

IP : 34.239 .49 .27

26 апреля 2023 г., $06: 50: 47$

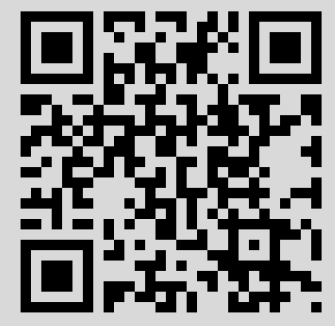


УДК 512.54

\section{О НОРМАЛИЗАТОРАХ НЕКОТОРЫХ \\ КЛАССОВ ПОДГРУПП В ГРУППАХ КОС}

\section{В. Н. Безверхний, И. В. Добрынина}

В данной работе изучаются нормализаторы некоторых классов подгрупп группы кос $B_{n+1}$.

Библиографиия: 8 названий.

Группа кос $B_{n+1}$ задается образующими $\sigma_{1}, \ldots, \sigma_{n}$ и определяющими соотоношениями:

$$
\begin{aligned}
& \sigma_{i} \sigma_{i+1} \sigma_{i}=\sigma_{i+1} \sigma_{i} \sigma_{i+1}, \quad i=1, \ldots, n-1 \\
& \sigma_{i} \sigma_{j}=\sigma_{j} \sigma_{i}, \quad i, j=1, \ldots, n, \quad|i-j|>1
\end{aligned}
$$

Известно существование гомоморфизма группы $B_{n+1}$ в группу подстановок $S_{n+1}$, отображающего $\sigma_{i}$ в транспозицию $(i, i+1), i=1, \ldots, n$. Коса, реализующая единичную подстановку, называется крашеной. Подгруппа крашеных кос группы $B_{n+1}$ обозначается через $R_{n+1}$, ее образуюшие - косы $\sigma_{j-1} \sigma_{j-2} \ldots \sigma_{i}^{2} \ldots \sigma_{j-2}^{-1} \sigma_{j-1}^{-1}(1 \leqslant i<j \leqslant n+$ $1)$ - через $s_{i, j}$. Через $U_{j}, j=1, \ldots, n$, обозначается подгрупша из $R_{n+1}$, порожденная свободными образуюшими $s_{1, j+1}, s_{2, j+1}, \ldots, s_{j, j+1}$. Известно, что всякая крашенная коса из $R_{n+1}$ однозначно представима в виде произведения $F_{1} F_{2} \ldots F_{n}$, где $F_{i} \in U_{i}$, $i=1, \ldots, n$.

Пусть $U_{m-1}$ - свободная группа с образующими $s_{1, m}, s_{2, m}, \ldots s_{m-1, m}$. Для любых $r, p, 1 \leqslant r<p<m$, любого $i, 1 \leqslant i<m$, и любого $\varepsilon= \pm 1$ справедливы соотношения [1]

$$
s_{r, p}^{-\varepsilon} s_{i, m} s_{r, p}^{\varepsilon}=\left\{\begin{array}{c}
s_{i, m}, \quad \text { если } r<p<i<m \text { или } i<r<p<m, \\
\left(s_{r, m} s_{p, m}\right)^{\varepsilon} s_{i, m}\left(s_{r, m} s_{p, m}\right)^{-\varepsilon}, \quad \text { если } r<i=p<m \\
\text { или } r=i<p<m, \\
\left(s_{r, m} s_{p, m}\right)^{\varepsilon}\left(s_{p, m} s_{r, m}\right)^{-\varepsilon} s_{i, m}\left(s_{p, m} s_{r, m}\right)^{\varepsilon}\left(s_{r, m} s_{p, m}\right)^{-\varepsilon}, \\
\text { если } r<i<p<m .
\end{array}\right.
$$

В данной работе изучаются нормализаторы некоторых классов подгрупп группы $B_{n+1}$.

Работа выполнена при финансовой поддержке Российского фонда фундаментальных исследований, грант № 00-01-00767. 
УТВЕРЖДЕНИЕ [2]. Пусть $H$ - подгруппа конечного индекса в $G$. порохсдена тогда и только тогда, когда $G$ конечно порохсдена.

Используя данное утверждение, вопрос о конечной порожденности подгруппы $H$ в $B_{n+1}$ сводится к вопросу о конечной порожденности подгруппы $H \cap R_{n+1}$. С помощью методов работы [3] доказывается

Теорема 1. Пусть $H$ - конечно порохсденая подгруппа группы $B_{n+1}$. Тогда существует алгоритм, строящий порождающие подгруппы $H \cap R_{n+1}$.

ДОКАЗАТЕЛЬСТВо. Положим $m_{i, j}=\sigma_{i-1} \sigma_{i-2} \ldots \sigma_{j}$ для $j<i$ и $m_{i, j}=1$ в противном случае. Тогда множество $\Lambda_{n+1}=\left\{\prod_{i=1}^{n+1} m_{i, j} \mid 1 \leqslant j \leqslant i\right\}$ образует шрейерову систему представителей смежных классов $B_{n+1}$ по $R_{n+1}$, а всякий элемент $w \in B_{n+1}$ можно представить в виде $w=\widetilde{w} \bar{w}$, где $\bar{w} \in R_{n+1}, \widetilde{w} \in \Lambda_{n+1}[4]$.

Пусть $S_{n+1}=\left\langle b_{1}, \ldots, b_{m} ; A_{1}\left(b_{\mu}\right), \ldots, A_{r}\left(b_{\mu}\right)\right\rangle$ - представление группы подстановок, заданное таблицей Кэли, где $m=(n+1) !$, причем между элементами $b_{i}, i=1, \ldots, m$, и шрейеровыми представителями из $\Lambda_{n+1}$ существует взаимно-однозначное соответствие, которое устанавливается эффективно; $R_{n+1}=\left\langle s_{i, j}(1 \leqslant i<j \leqslant n+1) ; B_{1}\left(s_{i, j}\right), \ldots\right.$ $\left.\ldots, B_{k}\left(s_{i, j}\right)\right\rangle$ - представление групшы крашеных кос, где $B_{t}\left(s_{i, j}\right), t=1, \ldots, k,-$ соотношение Бурау (1).

Рассмотрим группу кос

$$
\begin{aligned}
B_{n+1}= & \left\langle b_{1}, \ldots, b_{m}, s_{i, j}(1 \leqslant i<j \leqslant n+1) ; B_{1}\left(s_{i, j}\right), \ldots, B_{k}\left(s_{i, j}\right),\right. \\
& \left.A_{r}\left(b_{\mu}\right)=W_{r}\left(s_{i, j}\right), b_{l} s_{i, j} b_{l}^{-1}=W_{l i j}\left(s_{p, t}\right), b_{l}^{-1} s_{i, j} b_{l}=V_{l i j}\left(s_{p, t}\right)\right\rangle,
\end{aligned}
$$

где соотношения $A_{r}\left(b_{\mu}\right)=W_{r}\left(s_{i, j}\right), b_{l} s_{i, j} b_{l}^{-1}=W_{l i j}\left(s_{p, t}\right), b_{l}^{-1} s_{i, j} b_{l}=V_{l i j}\left(s_{p, t}\right)$, получаются подстановкой вместо $b_{j}$ соответствующего шрейерова представителя и перезаписи полученных слов в образующих $R_{n+1}$ с помощью переписывающего процесса Рейдемейстера-Шрейера [2].

Рассмотрим конечно-порожденную подгруппу $H \subset B_{n+1}$. Пусть $\alpha_{1} y_{1}, \ldots, \alpha_{k_{0}} y_{k_{0}}$, $y_{k_{0}+1}, \ldots, y_{k_{1}}$ - образуюшие $H$, где $\alpha_{i} \in S_{n+1}, i=1, \ldots, k_{0}, y_{j} \in R_{n+1}, j=1, \ldots, k_{1}$. Пусть $\varphi$ - гомоморфизм групшы $B_{n+1}$ на $S_{n+1}: \varphi\left(B_{n+1}\right)=S_{n+1}$, определяемьй следуюшим образом: $\forall s_{i, j} \in R_{n+1}, 1 \leqslant i<j \leqslant n+1, \varphi\left(s_{i, j}\right)=1, \forall l, l=1, \ldots, m$, $\varphi\left(b_{l}\right)=b_{l}$. Тогда $\varphi(H)=g r\left(\alpha_{1}, \ldots, \alpha_{k_{0}}\right), \varphi(H) \subset S_{n+1}$. Вьпишем все элементы подгрупшы $\varphi(H): 1, \alpha_{1}^{\prime}, \ldots, \alpha_{k_{0}^{\prime}}^{\prime}$, где $\alpha_{i}^{\prime}=\omega_{i}\left(\alpha_{p}\right), \omega_{i}\left(\alpha_{p}\right)-$ слова в образующих $\left\{\alpha_{j}\right\}, j=1, \ldots, k_{0}$, подгрупшы $\varphi(H) \subset S_{n+1}$. Используя таблицу умножения Кэли, получим представление подгруппы $\varphi(H) \subset S_{n+1}$, задаваемой также таблицей Кэли: $\varphi(H)=\left\langle\alpha_{0}, \alpha_{1}^{\prime}, \ldots, \alpha_{k_{0}^{\prime}}^{\prime} ; \psi_{1}, \ldots, \psi_{\alpha}\right\rangle, \psi_{i}=\psi_{i}\left(\alpha_{b}^{\prime}\right), i=1, \ldots, \alpha, \alpha_{0}=1$.

В качестве образующих подгруппы $H$ возьмем элементы

$$
\begin{gathered}
\alpha_{1}^{\prime} y_{1}^{\prime}, \alpha_{2}^{\prime} y_{2}^{\prime}, \ldots, \alpha_{k_{0}^{\prime}}^{\prime} y_{k_{0}^{\prime}}^{\prime}, y_{k_{0}^{\prime}+1}^{\prime}, \ldots, y_{k_{1}^{\prime}}^{\prime}, \quad k_{1}^{\prime}=k_{1}-k_{0}+k_{0}^{\prime}, \\
\forall i, \quad 1 \leqslant i \leqslant k_{1}-k_{0}, \quad y_{k_{0}^{\prime}+i}^{\prime}=y_{k_{0}+i}, \quad \alpha_{j}^{\prime} y_{j}^{\prime}=\omega_{j}\left(\alpha_{p} y_{p}\right), \quad j=1, \ldots, k_{0}^{\prime} .
\end{gathered}
$$

Последнее всегда можно получить, используя преобразования (2).

Обозначим множество элементов $\left\{y_{k_{0}^{\prime}+j}^{\prime}\right\}, j=1, \ldots, k_{1}-k_{0}$, через $Y_{0}$. Соотношениям $\psi_{i}, i=1, \ldots, \alpha$, подгруппы $\varphi(H)$ в групе $B_{n+1}$ будут соответствовать соотношения $\psi_{i}=N_{i}, i=1, \ldots, \alpha, N_{i} \in R_{n+1}$. 
В подгрупше $H$ рассмотрим слова $\psi_{i}\left(\alpha_{j}^{\prime} y_{j}^{\prime}\right)$, полученные подстановкой в $\psi_{i}\left(\alpha_{j}^{\prime}\right)$ вместо $\alpha_{j}^{\prime}$ образующих $\alpha_{j}^{\prime} y_{j}^{\prime}$ подгруппы $H$. Используя соотношения групшы $B_{n+1}$, заданной представлением (2), получим $\psi_{i}\left(\alpha_{j}^{\prime} y_{j}^{\prime}\right)=\psi_{i}\left(\alpha_{j}^{\prime}\right) T_{i}\left(s_{i, j}\right)$, где $\psi_{i}\left(\alpha_{j}^{\prime}\right) \in S_{n+1}, T_{i}\left(s_{i, j}\right) \in$ $R_{n+1}$. Присоединим к $Y_{0}$ все элементы вида $N_{i} T_{i} \in R_{n+1}, i=1, \ldots, \alpha$, получим множество $Y_{1}$. На образующих $\left\{\alpha_{j}^{\prime} y_{j}^{\prime}\right\}, j=1, \ldots, k_{0}^{\prime}$, из (3) подгруппы $H$ рассмотрим всевозможные произведения вида $\left(\alpha_{i}^{\prime} y_{i}^{\prime}\right)^{\varepsilon_{i}}\left(\alpha_{j}^{\prime} y_{j}^{\prime}\right)^{\varepsilon_{j}}, \varepsilon_{i}= \pm 1, \varepsilon_{j}= \pm 1, i, j \in\left\{1, \ldots, k_{0}^{\prime}\right\}$. Каждое из этих произведений приведем к виду $\left(\alpha_{i}^{\prime} y_{i}^{\prime}\right)^{\varepsilon_{i}}\left(\alpha_{j}^{\prime} y_{j}^{\prime}\right)^{\varepsilon_{j}}=\alpha_{i j}^{\varepsilon_{i j}} y_{i j}^{\varepsilon_{i j}}$. Те из соотношений, у которых $\alpha_{i j}^{\varepsilon_{i} \varepsilon_{j}}=1\left(\varepsilon_{i} \varepsilon_{j}=\varepsilon_{i j}\right)$, присоединяем к $Y_{1}$; получим множество $Y_{2}$. Затем для каждого $\alpha_{i j}^{\varepsilon_{i j}} y_{i j}^{\varepsilon_{i j}}$ с $\alpha_{i j}^{\varepsilon_{i j}} \neq 1$ из множества (3) берем элемент $\alpha_{s}^{\prime} y_{s}^{\prime}$ такой, что $\alpha_{s}^{\prime}=\alpha_{i j}^{\varepsilon_{i j}}$, и составляем произведения $y_{s, i j}=y_{s}^{-1} y_{i j}^{\varepsilon_{i j}}, y_{i j, s}=\alpha_{i j}^{\varepsilon_{i j}} y_{i j}^{\varepsilon_{i j}} y_{s}^{\prime-1} \alpha_{s}^{\prime-1}$, добавив которые к $Y_{2}$, получим множество $Y_{3}$.

Пусть $C=\operatorname{gr}\left(Y_{3}\right)$. Сопрягаем $C$ элементами $\left(\alpha^{\prime}{ }_{i} y_{i}^{\prime}\right)^{\varepsilon_{i}}, \varepsilon_{i}= \pm 1$, где $\alpha_{i}^{\prime} y_{i}^{\prime}$ принадлежит множеству (3). Получим подгруппы $C_{\alpha^{\prime}{ }_{i}{ }_{i}}=\left(\alpha^{\prime}{ }_{i} y_{i}^{\prime}\right)^{\varepsilon_{i}} C\left(\alpha^{\prime}{ }_{i} y^{\prime}{ }_{i}\right)^{-\varepsilon_{i}}, i=1, \ldots, k_{0}^{\prime}$, $\varepsilon_{i}= \pm 1$. Покажем, что $\forall i \exists j, 1 \leqslant i, j \leqslant k_{0}^{\prime}, C_{\alpha^{\prime-1}}=C_{\alpha^{\prime}{ }_{j}}$. Действительно, $C_{\alpha^{\prime}-1}=$ $\left(\alpha^{\prime}{ }_{i} y_{i}^{\prime}\right)^{-1} C\left(\alpha_{i}^{\prime} y_{i}^{\prime}\right)=\alpha_{i}^{\prime-1} y_{i}^{-\alpha_{i}} C y_{i}^{\alpha_{i}} \alpha_{i}^{\prime}$, где $y_{i}^{\alpha_{i}}=\alpha_{i}^{\prime} y_{i}^{\prime} \alpha_{i}^{\prime-1}, y_{i}^{-\alpha_{i}}=\alpha_{i}^{\prime} y_{i}^{\prime-1} \alpha_{i}^{\prime-1}$. Так как $\alpha_{i}^{\prime-1} \in \varphi(H)$, сушествует $\alpha_{j}^{\prime} \in \varphi(H)$ такое, что $\alpha_{j}^{\prime}=\alpha_{i}^{\prime-1}$. Поэтому

$$
C_{\alpha_{i}^{\prime-1}}=\alpha^{\prime}{ }_{j} y_{j}^{\prime}\left(y_{i}^{\prime \prime}\right)^{-1} C y_{i}^{\prime \prime} y_{j}^{\prime-1} \alpha^{\prime-1}=C_{\alpha^{\prime}{ }_{j}},
$$

где $\left(y_{i}^{\prime \prime}\right)^{-1}=y_{j}^{\prime-1} \alpha^{\prime-1} \alpha_{i}^{\prime-1} y_{i}^{-\alpha_{i}} \in C$.

Рассмотрим подгруппу $Y=\operatorname{gr}\left(C, C_{\alpha_{1}^{\prime}}, \ldots, C_{\alpha_{k_{0}^{\prime}}^{\prime}}\right)$ подгруппы $H$.

ЛЕмма 1. Подгруппа $Y$ является нормальным делителем подгруппь $H$.

ДокАЗАТЕЛЬСТВо очевидно.

Подгруппу $H$ в дальнейшем будем представлять таким образом: $H=\operatorname{gr}\left(\alpha_{1}^{\prime} y_{1}^{\prime}, \ldots\right.$ $\left.\ldots, \alpha_{k_{0}^{\prime}} y_{k_{0}}^{\prime}, Y\right)$.

ЛЕмма 2. Каждый әлемент $w \in H$ единственным способом может быть представлен в виде $w=\alpha_{i}^{\prime} y_{i}^{\prime} y$, где $\alpha_{i}^{\prime} y_{i}^{\prime}$ принадлехсат (3), $y \in Y$.

ДокАЗАТЕЛЬСТВо очевидно.

Лемма 3. Имеем $H \cap R_{n+1}=Y$.

ДокАЗАТЕЛЬСтво непосредственно следует из леммы 2.

Теорема доказана.

СлЕДСТВИЕ 1. Централизатор конечного множества әлементов из $R_{n+1}$ конечно порохсден в $R_{n+1}$. Существует алгоритм, строящий образующие этого иентрализатора.

ДокАЗАТЕЛЬСтво следует из [5], утверждения и теоремы 1.

СлЕДСТВИЕ 2. Нормализатор конечного множества әлементов из $R_{n+1}$ конечно порохсден в $R_{n+1}$. Существует алгоритм, строящий образующие этого нормализатора. 
ДокАЗАТЕЛЬСТво следует из [5], утверждения и теоремы 1.

СлЕДСТВИЕ 3. Нормализатор конечно-порожденной подгруппы в $R_{3}$ конечно порожден. Существует алгоритм, строящий образующие этого нормализатора.

ДоказАтЕЛьство. Известно [6], что $B_{3}$ изоморфнагруппе $G=\left\langle x, y ; x^{3}=y^{2}\right\rangle$. Рассмотрим группу $G^{*}=G /\left\langle y^{2}\right\rangle=\left\langle x, y ; x^{3}, y^{2}\right\rangle$. Учитывая, что $y^{2}=1, x^{2}=x^{-1}, x^{3}=1$, каждьй элемент $G^{*}$ может быть однозначно записан через элементы $x, x^{-1}, y$ и длина его определена как число указанных элементов в данном слове. Любое конечное множество слов с помощью преобразований, аналогичных нильсеновским, может быть приведено к множеству с наименьшей суммарной длиной, позволяющему однозначно перезаписьвать элементы, порожденные данным множеством (при этом следует заметить, что в записи элементов исключаются степени образующих, равные единице). Используя процедуру построения нормализатора конечно-порожденной подгрупшы в свободной групе [2] и учитьвая разрешимость проблемы вхождения в свободном произведении групп [7], получаем конечную порожденность нормализатора конечно-порожденной подгрупшы в $G^{*}$ и алгоритм построения данного нормализатора. Таким образом, нормализатор конечно-порожденной подгрупы из $B_{3}$ конечно порожден в $B_{3}$ и существует алгоритм, вьписьвающий его образующие. Отсюда по теореме 1 получаем утверждение данного следствия.

Теорема 2. Пусть $G=F_{1} \times F_{2}$ - прямое произведение двух свободных групп ранга 2. Пусть $H$ - конечно-порожсденная подгруппа из $G$. Тогда нормализатор $N_{G}(H)$ конечно порожден.

ДОКАЗАТЕЛЬСТВО. Пусть

$$
H=\left\langle a_{1} b_{1}, \ldots, a_{n} b_{n}, a_{n+1}, \ldots, a_{k}, b_{n+1}, \ldots, b_{t}\right\rangle .
$$

Будем считать, что $a_{i}, i=1, \ldots, k,-$ свободные образуюшие. Допустим, что $N_{G}(H)$ бесконечно порожден. Рассмотрим подгруппы $L=\left\langle a_{1}, a_{2}, \ldots, a_{n}, a_{n+1}, \ldots, a_{k}\right\rangle, K=$ $\left\langle b_{1}, b_{2}, \ldots, b_{n}, b_{n+1}, \ldots, b_{t}\right\rangle$, являющиеся проекциями $H$ на $F_{1}$ и на $F_{2}$ соответственно. Возможны следующие случаи.

1. Случай $n \geqslant 2$. Пусть $Z \in N_{G}(H)$ и $Z=A B$, где $A \in F_{1}, B \in F_{2}, Z$ - образующий $N_{G}(H)$. Выберем $Z$ таким, чтобы в смежном классе $H Z H$ элемент $B$ имел наименьшую длину. Это означает, что $B$ имеет наименьшую длину в смежном классе $K B K$. Преобразуем $\left\{b_{j}\right\}, j=1, \ldots, t$, к нильсеновскому множеству. Тогда $|B| \leqslant 1 / 2$ длины максимального нильсеновского образующего. Следовательно, элементов $B$ - конечное число.

Известно, что $\left|N_{F_{1}}(L) / L\right|<\infty[2]$. Пусть $r_{1}, r_{2}, \ldots, r_{m}$ - представители смежных классов $N_{F_{1}}(L)$ по $L$. Тогда образующий $Z=A B$ из $N_{G}(H)$ можно преобразовать к виду $r_{i} A_{l} B_{j}$, где $B_{j}$ - представитель из $K B K$, имеющий наименьшую длину, $A_{l} \in L, r_{i}-$ некоторый указанньй выше представитель. Так как элементов $r_{i}$ и $B_{j}-$ конечное число, то, исключая одинаковые $r_{i}$, а затем $B_{j}$ (либо наоборот) из $\left\{r_{i} A_{l} B_{j}\right\}$, мы получим конечное число образующих вида $r_{i} A_{l} B_{j}$ и бесконечное число $A_{l}^{\prime}$ таких, что $A_{l}^{\prime} \in N_{G}(H)$ и $A_{l}^{\prime} \in L$. Тогда для $i=1, \ldots, n$ имеем: $A_{l}^{\prime-1} a_{i} A_{l}^{\prime} b_{i} \in H$ и $A_{l}^{\prime-1} a_{i} A_{l} a_{i}^{-1} \in H$ $\left(A_{l}^{\prime-1} a_{i} A_{l}^{\prime} a_{i}^{-1}=A_{l}^{\prime-1} a_{i} A_{l}^{\prime} b_{i}\left(a_{i} b_{i}\right)^{-1}\right)$. А так как $A_{l}^{\prime-1} a_{i} A_{l}^{\prime} a_{i}^{-1} \in F_{1}$, то $A_{l}^{\prime-1} a_{i} A_{l}^{\prime} a_{i}^{-1} \in$ $H \cap F_{1}$. Рассмотрим следуюшие подгрупшы: $M=\left\langle a_{1}, \ldots, a_{n}\right\rangle, N=\left\langle a_{n+1}, \ldots, a_{k}\right\rangle$, 
$N^{M}$ - нормальное замыкание $N$ с помощью $M$. Тогда $H \cap F_{1}=N^{M}$ и $A_{l}^{\prime-1} a_{i} A_{l}^{\prime} a_{i}^{-1}=$ $A_{l_{1}}$, где $A_{l_{1}} \in N^{M}$. Элемент $A_{l}^{\prime}$ можем представить как $A_{l}^{\prime}=A_{l_{2}} A_{l_{3}}$, где $A_{l_{2}} \in M$, а $A_{l_{3}} \in N^{M}$. Имеем $A_{l_{3}}^{-1} A_{l_{2}}^{-1} a_{i} A_{l_{2}} A_{l_{3}} a_{i}^{-1}=A_{l_{1}}$; отсюда $A_{l_{2}}^{-1} a_{i} A_{l_{2}} a_{i}^{-1}=A_{l_{4}}$, где $A_{l_{4}} \in N^{M}$. Так как $M \cap N^{M}=E$, где $E$ - единичная подгруппа, то

$$
A_{l_{2}}^{-1} a_{i} A_{l_{2}}=a_{i}, \quad i=1, \ldots, n .
$$

Так как $a_{i}$ - свободные образуюшие, то $A_{l_{2}}=1, A_{l}^{\prime}=A_{l_{3}}$. Таким образом, $A_{l}^{\prime} \in H$.

2. Случай $n=1$.

a) Подгрупша $H$ имеет вид $\left\langle a_{1} b_{1}, a_{2}, \ldots, a_{k}, b_{2}, \ldots, b_{t}\right\rangle$.

Из (4) имеем

$$
A_{l_{2}}^{-1} a_{1} A_{l_{2}}=a_{1} .
$$

Следовательно, $A_{l_{2}}=a_{1}^{k_{l}}$ и элемент $A_{l}^{\prime}$ примет вид $A_{l}^{\prime}=a_{1}^{k_{l}} A_{l_{3}}$, где $A_{l_{3}} \in N^{M}, M=$ $\left\langle a_{1}\right\rangle$. Так как $a_{1}^{-1} a_{1} b_{1} a_{1}=a_{1} b_{1}, a_{1}^{-1} b_{j} a_{1}=b_{j}, j=2, \ldots, t$, то $a_{1} \in N_{G}(H)$. Поэтому в записи элементов $A_{l}^{\prime}$ мы можем исключить элементы $a_{1}^{k_{l}}$ и получить элементы $A_{l_{3}} \in$ $N_{G}(H)$.

б) Подгруппа $H$ имеет вид $\left\langle a_{1} b_{1}, b_{2}, \ldots, b_{k}\right\rangle$. Пусть $Z=A B \in N_{G}(H)$. Из п. 1 следует, что элементов $B$ можно взять конечное число. Если элементов $Z$, порождающих $N_{G}(H)$, бесконечно много, то, исключая одинаковые $B$, мы получим бесконечное число элементов $A$, удовлетворяюших соотношению $A^{-1} a_{1} A=a_{1}$. Следовательно, $A=x^{t}$, $a=x^{p}$, где $x \in F_{1}$. И так как $x \in N_{G}(H)$, отсюда следует, что нормализатор подгруппы $H$ конечно порожден.

в) Подгруппа $H$ имеет вид $\left\langle a_{1} b_{1}, a_{2}, \ldots, a_{k}\right\rangle$. Доказательствопроводится аналогично пункту $2 \mathrm{a}$.

г) Подгрупша $H$ имеет вид $\left\langle a_{1} b_{1}\right\rangle$. Пусть $Z \in N_{G}(H)$ и $Z=A B$, где $A \in F_{1}, B \in F_{2}$. Тогда $A^{-1} a_{1} A=a_{1}$ и $B^{-1} b_{1} B=b_{1}$. Следовательно, $A=x^{s}, a_{1}=x^{t}, x \in F_{1}$, и $B=y^{m}, b_{1}=y^{p}, y \in F_{2} ; x, y$ не являются истинньми степенями. Поэтому $N_{G}(H)=$ $\langle x\rangle \times\langle y\rangle$.

3. Подгруппа $H$ имеет вид $\left\langle a_{1}, a_{2}, \ldots, a_{k}, b_{1}, \ldots, b_{t}\right\rangle$. Пусть $L=\left\langle a_{1}, \ldots, a_{k}\right\rangle, K=$ $\left\langle b_{1}, \ldots, b_{t}\right\rangle$. Тогда $N_{G}(H)=N_{F_{1}}(L) N_{F_{2}}(K)$. Так как $N_{F_{1}}(L), N_{F_{2}}(K)$ конечно порождены, то $N_{G}(H)$ конечно порожден.

Теорема доказана.

Рассмотрим в $R_{5}$ подгрупп $\Pi$, порожденную элементами $\sigma_{1}^{4}, \sigma_{2}^{4}, \sigma_{4}^{2}, d$, где $d=\sigma_{4} \sigma_{3} \times$ $\sigma_{2} \sigma_{1}^{2} \sigma_{2} \sigma_{3} \sigma_{4}$. Известно [6], что $\Pi=\left\langle\sigma_{1}^{4}, \sigma_{2}^{4}\right\rangle \times\left\langle\sigma_{4}^{2}, d\right\rangle-$ прямое произведение двух свободных групп ранга 2 . Обозначим через $\tau$ перезапись слова $X\left(\sigma_{1}, \ldots, \sigma_{4}\right) \in R_{5}$ в образуюших $s_{i, j} ; \tau(d)=s_{1,5} s_{2,5} s_{3,5} s_{4,5}$.

Теорема 3. Имеем $N_{R_{5}}(\Pi)=\left\langle\Delta^{2}, \Delta^{\prime 2}, \Pi\right\rangle$, где $\Delta=\sigma_{1} \sigma_{2} \sigma_{3} \sigma_{4} \sigma_{1} \sigma_{2} \sigma_{3} \sigma_{1} \sigma_{2} \sigma_{1}$, $\Delta^{\prime}=\sigma_{2} \sigma_{1} \sigma_{2}$.

ДокАЗАТЕЛЬСтво. Пусть $Z \in N_{R_{5}}(\Pi)$. Тогда $Z$ переводит любое слово из П снова в слово из П. В частности, $Z$ удовлетворяет системе

$$
\begin{aligned}
Z^{-1} \sigma_{1}^{4} \sigma_{4}^{2} Z & =\varphi_{1}\left(\sigma_{1}^{4}, \sigma_{2}^{4}\right) \psi_{1}\left(\sigma_{4}^{2}, d\right), \\
Z^{-1} \sigma_{2}^{4} d Z & =\varphi_{2}\left(\sigma_{1}^{4}, \sigma_{2}^{4}\right) \psi_{2}\left(\sigma_{4}^{2}, d\right),
\end{aligned}
$$

где $\varphi_{i}\left(\sigma_{1}^{4}, \sigma_{2}^{4}\right) \psi_{i}\left(\sigma_{4}^{2}, d\right)$ - слова из $\Pi, i=1,2$.

Представим $Z$ в виде $Z=F_{1} F_{2} F_{3} F_{4}$, где $F_{j} \in U_{j}$. 
Лемма 4. В $R_{5}$ система (5) эквивалентна системе

$$
\begin{gathered}
F_{2}^{-1} F_{1}^{-1} \sigma_{1}^{4} F_{1} F_{2}=\varphi_{1}\left(\sigma_{1}^{4}, \sigma_{2}^{4}\right), \\
F_{2}^{-1} F_{1}^{-1} \sigma_{2}^{4} F_{1} F_{2}=\varphi_{2}\left(\sigma_{1}^{4}, \sigma_{2}^{4}\right), \\
\left(F_{2}^{-1} F_{1}^{-1} \sigma_{1}^{-4} F_{1} F_{2}\right) F_{3}^{-1}\left(F_{2}^{-1} F_{1}^{-1} \sigma_{1}^{4} F_{1} F_{2}\right) F_{3}=1, \\
\left(F_{2}^{-1} F_{1}^{-1} \sigma_{2}^{-4} F_{1} F_{2}\right) F_{3}^{-1}\left(F_{2}^{-1} F_{1}^{-1} \sigma_{2}^{4} F_{1} F_{2}\right) F_{3}=1, \\
\left(F_{2}^{-1} F_{1}^{-1} \sigma_{1}^{-4} F_{1} F_{2}\right) F_{4}^{-1}\left(F_{2}^{-1} F_{1}^{-1} \sigma_{1}^{4} F_{1} F_{2}\right) F_{3}^{-1} \sigma_{4}^{2} F_{3} F_{4}=\psi_{1}\left(\sigma_{4}^{2}, d\right), \\
\left(F_{2}^{-1} F_{1}^{-1} \sigma_{2}^{-4} F_{1} F_{2}\right) F_{4}^{-1}\left(F_{2}^{-1} F_{1}^{-1} \sigma_{2}^{4} F_{1} F_{2}\right) F_{3}^{-1} d F_{3} F_{4}=\psi_{2}\left(\sigma_{4}^{2}, d\right),
\end{gathered}
$$

состоящей из систем (6), (7), (8).

ДокАЗАТЕльСтво. Подставим представление $Z=F_{1} F_{2} F_{3} F_{4}$ в систему (5) и преобразуем ее следуюшим образом:

$$
\begin{gathered}
\left(F_{2}^{-1} F_{1}^{-1} \sigma_{1}^{4} F_{1} F_{2}\right)\left(F_{2}^{-1} F_{1}^{-1} \sigma_{1}^{-4} F_{1} F_{2}\right) F_{3}^{-1}\left(F_{2}^{-1} F_{1}^{-1} \sigma_{1}^{4} F_{1} F_{2}\right) \\
\times F_{3}\left(F_{3}^{-1} F_{2}^{-1} F_{1}^{-1} \sigma_{1}^{-4} F_{1} F_{2} F_{3}\right) F_{4}^{-1}\left(F_{3}^{-1} F_{2}^{-1} F_{1}^{-1} \sigma_{1}^{4}\right. \\
\left.\times F_{1} F_{2} F_{3}\right) F_{3}^{-1} \sigma_{4}^{2} F_{3} F_{4}=\varphi_{1}\left(\sigma_{1}^{4}, \sigma_{2}^{4}\right) \psi_{1}\left(\sigma_{4}^{2}, d\right) \\
\left(F_{2}^{-1} F_{1}^{-1} \sigma_{2}^{4} F_{1} F_{2}\right)\left(F_{2}^{-1} F_{1}^{-1} \sigma_{2}^{-4} F_{1} F_{2}\right) F_{3}^{-1}\left(F_{2}^{-1} F_{1}^{-1} \sigma_{2}^{4} F_{1} F_{2}\right) \\
\times F_{3}\left(F_{3}^{-1} F_{2}^{-1} F_{1}^{-1} \sigma_{2}^{-4} F_{1} F_{2} F_{3}\right) F_{4}^{-1}\left(F_{3}^{-1} F_{2}^{-1} F_{1}^{-1} \sigma_{2}^{4}\right. \\
\left.\times F_{1} F_{2} F_{3}\right) F_{3}^{-1} d F_{3} F_{4}=\varphi_{2}\left(\sigma_{1}^{4}, \sigma_{2}^{4}\right) \psi_{2}\left(\sigma_{4}^{2}, d\right) .
\end{gathered}
$$

В силу однозначности представления всякого элемента $y \in R_{n+1}$ в виде $y=y_{1} y_{2} \ldots y_{n}$, где $y_{i} \in U_{i}$, из (9) получаем системы (6), (7) и

$$
\begin{aligned}
& \left(F_{3}^{-1} F_{2}^{-1} F_{1}^{-1} \sigma_{1}^{-4} F_{1} F_{2} F_{3}\right) F_{4}^{-1}\left(F_{3}^{-1} F_{2}^{-1} F_{1}^{-1} \sigma_{1}^{4} F_{1} F_{2} F_{3}\right) \\
& \times F_{3}^{-1} \sigma_{4}^{2} F_{3} F_{4}=\psi_{1}\left(\sigma_{4}^{2}, d\right), \\
& \left(F_{3}^{-1} F_{2}^{-1} F_{1}^{-1} \sigma_{2}^{-4} F_{1} F_{2} F_{3}\right) F_{4}^{-1}\left(F_{3}^{-1} F_{2}^{-1} F_{1}^{-1} \sigma_{2}^{4} F_{1} F_{2} F_{3}\right) \\
& \times F_{3}^{-1} d F_{3} F_{4}=\psi_{2}\left(\sigma_{4}^{2}, d\right) .
\end{aligned}
$$

Используя (7), преобразуем (10) к виду (8). Обратное утверждение очевидно.

Лемма 5 [6]. Пусть $H_{1}, H_{2}-$ две конечно-порожденные подгруппы из $\left\langle\sigma_{1}^{4}, \sigma_{2}^{4}\right\rangle$ и ни одна из них несопряжена с подгруппами вида $\left\langle\sigma_{j}^{4 t}\right\rangle, j=1,2, t \in \mathbb{Z}$. Если при этом существует $Z \in R_{3}$ такое, что $H_{1} Z=Z H_{2}$ в $R_{3}$, то $Z=\Delta^{\prime 2 k} S\left(\sigma_{1}^{4}, \sigma_{2}^{4}\right)$, $k \in \mathbb{Z}$, әде $\Delta^{\prime}$ - фундаментальный әлемент $B_{3}$.

Лемма 6 [6]. Если $Z^{\prime}=F_{1} F_{2} \quad\left(F_{i} \in U_{i}, i=1,2\right)$ удовлетворяет системе (6), mo $Z^{\prime}=\Delta^{\prime 2 k} L_{1}\left(\sigma_{1}^{4}, \sigma_{2}^{4}\right), \quad k \in \mathbb{Z}$.

Лемма 7. Если $F_{3} \in U_{3}$ является решением системы $(7)$, по $\tau\left(F_{3}\right)=\left(s_{1,4} \times\right.$ $\left.s_{2,4} s_{3,4}\right)^{k}, \quad k \in \mathbb{Z}$. 
ДокАЗАтЕльство. Очевидно, коса $F_{3}=\left(\sigma_{3} \sigma_{2} \sigma_{1}^{2} \sigma_{2} \sigma_{3}\right)^{k}$ является решением системы $(7)$, причем $\tau\left(F_{3}\right)=\left(s_{1,4} s_{2,4} s_{3,4}\right)^{k}, k \in \mathbb{Z}$. Если существует коса $X \in U_{3}$, являющаяся решением (7) и $X \neq F_{3}$, то в силу принадлежности $X$ и $F_{3}$ свободной групе $U_{3}$ в $B_{4}$ содержится прямое произведение двух свободных групп ранга $2\left\langle F_{2}^{-1} F_{1}^{-1} \sigma_{1}^{4} F_{1} F_{2}\right.$, $\left.F_{2}^{-1} F_{1}^{-1} \sigma_{2}^{4} F_{1} F_{2}\right\rangle \times\left\langle X, F_{3}\right\rangle$, что противоречит [8].

ЛЕмма 8. Справедливо равенство

$$
\tau(d)\left(s_{1,4} s_{2,4} s_{3,4}\right)=\left(s_{1,4} s_{2,4} s_{3,4}\right) \tau(d) \quad \text { в } R_{5}
$$

Используя непосредственно равенства (1), получаем доказательство данного утверждения.

Лемма 9. В $R_{5}$ имеет место соотношение

$$
\left(s_{1,4} s_{2,4} s_{3,4}\right)^{-k} s_{4,5}\left(s_{1,4} s_{2,4} s_{3,4}\right)^{k}=(\tau(d))^{k} s_{4,5}(\tau(d))^{-k}, \quad k \in \mathbb{Z} .
$$

ДоКАЗАТЕЛЬСТВО проводим индукцией по $k$, используя соотношения $(1)$ и лемму 8.

Лемма 10. Если $F_{4} \in U_{4}$ удовлетворяет системе $(8)$, то $F_{4}=F_{4}\left(\sigma_{4}^{2}, d\right)$.

ДокАЗАТЕЛЬСТво. Обозначим через $\left|F_{4}\right|$ длину слова $F_{4} \in U_{4}$ в свободных образующих $s_{4,5}, s_{3,5}, s_{2,5}, \tau(d)$. Возьмем $k \in \mathbb{N}$ такое, что $k$ больше $3\left|F_{4}\right|$. Возведем левую и правую части каждого из равенств $(5)$ в $k$-ю степень. Учитывая представление $Z$ в виде $Z=F_{1} F_{2} F_{3} F_{4}$, имеем

$$
\begin{aligned}
F_{4}^{-1} F_{3}^{-1} F_{2}^{-1} F_{1}^{-1} \sigma_{1}^{4 k} \sigma_{4}^{2 k} F_{1} F_{2} F_{3} F_{4} & =\varphi_{1}^{k}\left(\sigma_{1}^{4}, \sigma_{2}^{4}\right) \psi_{1}^{k}\left(\sigma_{4}^{2}, d\right), \\
F_{4}^{-1} F_{3}^{-1} F_{2}^{-1} F_{1}^{-1} \sigma_{2}^{4 k} d^{k} F_{1} F_{2} F_{3} F_{4} & =\varphi_{2}^{k}\left(\sigma_{1}^{4}, \sigma_{2}^{4}\right) \psi_{2}^{k}\left(\sigma_{4}^{2}, d\right)
\end{aligned}
$$

Проводя рассуждения, изложенные в лемме 4, легко показать, что система (11) эквивалентна системе

$$
\begin{gathered}
F_{2}^{-1} F_{1}^{-1} \sigma_{1}^{4 k} F_{1} F_{2}=\varphi_{1}^{k}\left(\sigma_{1}^{4}, \sigma_{2}^{4}\right) \\
F_{2}^{-1} F_{1}^{-1} \sigma_{2}^{4 k} F_{1} F_{2}=\varphi_{2}^{k}\left(\sigma_{1}^{4}, \sigma_{2}^{4}\right) . \\
\left(F_{2}^{-1} F_{1}^{-1} \sigma_{1}^{-4 k} F_{1} F_{2}\right) F_{3}^{-1}\left(F_{2}^{-1} F_{1}^{-1} \sigma_{1}^{4 k} F_{1} F_{2}\right) F_{3}=1, \\
\left(F_{2}^{-1} F_{1}^{-1} \sigma_{2}^{-4 k} F_{1} F_{2}\right) F_{3}^{-1}\left(F_{2}^{-1} F_{1}^{-1} \sigma_{2}^{4 k} F_{1} F_{2}\right) F_{3}=1, \\
\left(F_{2}^{-1} F_{1}^{-1} \sigma_{1}^{-4 k} F_{1} F_{2}\right) F_{4}^{-1}\left(F_{2}^{-1} F_{1}^{-1} \sigma_{1}^{4 k} F_{1} F_{2}\right) \\
\times F_{3}^{-1} \sigma_{4}^{2 k} F_{3} F_{4}=\psi_{1}^{k}\left(\sigma_{4}^{2}, d\right), \\
\left(F_{2}^{-1} F_{1}^{-1} \sigma_{2}^{-4 k} F_{1} F_{2}\right) F_{4}^{-1}\left(F_{2}^{-1} F_{1}^{-1} \sigma_{2}^{4 k} F_{1} F_{2}\right) \\
\times F_{3}^{-1} d^{k} F_{3} F_{4}=\psi_{2}^{k}\left(\sigma_{4}^{2}, d\right),
\end{gathered}
$$

состоящей из систем (12), (13), (14).

Решения систем (12), (13) имеют вид, описанньй в леммах 6 и 7 . 
Используя лемму 8 и переписывающий процесс $\tau$, второе равенство из (14) перепишем следующим образом:

$$
\tau\left(F_{2}^{-1} F_{1}^{-1} \sigma_{2}^{-4 k} F_{1} F_{2}\right)\left(\tau\left(F_{4}\right)\right)^{-1} \tau\left(F_{2}^{-1} F_{1}^{-1} \sigma_{2}^{4 k} F_{1} F_{2}\right)(\tau(d))^{k} \tau\left(F_{4}\right)=\left(\tau\left(\psi_{2}\left(\sigma_{4}^{2}, d\right)\right)\right)^{k}
$$

и так как $\tau\left(F_{2}^{-1} F_{1}^{-1} \sigma_{2}^{4 k} F_{1} F_{2}\right)=\varphi_{2}^{k}\left(s_{1,2}^{2}, s_{2,3}^{2}\right)$ получаем

$$
\varphi_{2}^{-k}\left(s_{1,2}^{2}, s_{2,3}^{2}\right)\left(\tau\left(F_{4}\right)\right)^{-1} \varphi_{2}^{k}\left(s_{1,2}^{2}, s_{2,3}^{2}\right)(\tau(d))^{k} \tau\left(F_{4}\right)=\psi_{2}^{k}\left(s_{4,5}, \tau(d)\right)
$$

Слово $\tau\left(F_{4}\right)$ в несократимой форме в свободных образующих $s_{4,5}, s_{3,5}, s_{2,5}, \tau(d)$ представим в виде

$$
\tau\left(F_{4}\right)=\prod_{i=1}^{n} Q_{i}\left(s_{4,5}, \tau(d)\right) P_{i}\left(s_{2,5}, s_{3,5}\right)
$$

где $Q_{1}\left(s_{4,5}, \tau(d)\right)$ и $P_{n}\left(s_{2,5}, s_{3,5}\right)$ могут быть равны единице. Используя равенство $(1)$, имеем

$$
\begin{gathered}
\varphi_{2}^{-k}\left(s_{1,2}^{2}, s_{2,3}^{2}\right)\left(\prod_{i=n}^{1} P_{i}^{-1}\left(s_{2,5}, s_{3,5}\right) Q_{i}^{-1}\left(s_{4,5}, \tau(d)\right) \varphi_{2}^{k}\left(s_{1,2}^{2}, s_{2,3}^{2}\right)\right) \\
=\prod_{i=n}^{1} P_{i}^{\prime-1}\left(s_{1,5}, s_{2,5}, s_{3,5}\right) Q_{i}^{-1}\left(s_{4,5}, \tau(d)\right)
\end{gathered}
$$

где $P_{i}^{\prime-1}=\varphi_{2}^{-k}\left(s_{1,2}^{2}, s_{2,3}^{2}\right) P_{i}^{-1}\left(s_{2,5}, s_{3,5}\right) \varphi_{2}^{k}\left(s_{1,2}^{2}, s_{2,3}^{2}\right), i=1, \ldots, n, s_{1,5}$ можно записать как $s_{1,5}=\tau(d) s_{4,5}^{-1} s_{3,5}^{-1} s_{2,5}^{-1}$. Поэтому $P_{i}^{\prime-1}\left(s_{1,5}, s_{2,5}, s_{3,5}\right)=P_{i}^{\prime-1}\left(\tau(d) s_{4,5}^{-1} s_{3,5}^{-1} s_{2,5}^{-1}\right.$, $\left.s_{2,5}, s_{3,5}\right)$. Представим теперь $P_{i}^{\prime-1}$ в несократимой форме в свободных образующих $s_{2,5}, s_{3,5}, s_{4,5}, \tau(d)$ подгруппы $U_{4}$ :

$$
\begin{aligned}
P_{i}^{\prime-1}= & \left(\tau(d) s_{4,5}^{-1}\right)^{t_{i, 1}} P_{i, 1}^{\prime-1}\left(s_{2,5}, s_{3,5}\right)\left(\tau(d) s_{4,5}^{-1}\right)^{t_{i, 2}} P_{i, 2}^{\prime-1}\left(s_{2,5}, s_{3,5}\right) \cdots \\
& \cdots \times\left(\tau(d) s_{4,5}^{-1}\right)^{t_{i, m_{i}}} P_{i, m_{i}}^{\prime-1}\left(s_{2,5}, s_{3,5}\right)
\end{aligned}
$$

Подставим полученное выражение (16) в левую часть равенства (15). Имеем:

$$
\begin{aligned}
& \left(\prod_{i=n}^{1}\left(\tau(d) s_{4,5}^{-1}\right)^{t_{i, 1}} P_{i, 1}^{\prime-1}\left(s_{2,5}, s_{3,5}\right)\left(\tau(d) s_{4,5}^{-1}\right)^{t_{i, 2}}\right. \\
& \quad \times P_{i, 2}^{\prime-1}\left(s_{2,5}, s_{3,5}\right) \ldots\left(\tau(d) s_{4,5}^{-1}\right)^{t_{i, m_{i}} P_{i, m_{i}}^{\prime-1}\left(s_{2,5} s_{3,5}\right)} \\
& \left.\quad \times Q_{i}^{-1}\left(s_{4,5}, \tau(d)\right)\right)(\tau(d))^{k} \prod_{i=1}^{n} Q_{i}\left(s_{4,5}, \tau(d)\right) P_{i}\left(s_{2,5}, s_{3,5}\right) \\
& =\psi_{2}^{k}\left(s_{4,5}, \tau(d)\right)
\end{aligned}
$$


Обозначим

$$
\begin{aligned}
& \prod_{i=n}^{1}\left(\tau(d) s_{4,5}^{-1}\right)^{t_{i, 1}} P_{i, 1}^{\prime-1}\left(s_{2,5}, s_{3,5}\right)\left(\tau(d) s_{4,5}^{-1}\right)^{t_{i, 2}} \\
& \quad \times P_{i, 2}^{\prime-1}\left(s_{2,5}, s_{3,5}\right) \ldots\left(\tau(d) s_{4,5}^{-1}\right)^{t_{i, m_{i}} P_{i, m_{i}}^{\prime-1}\left(s_{2,5}, s_{3,5}\right)} \\
& \quad \times Q_{i}^{-1}\left(s_{4,5}, \tau(d)\right)=T \\
& \prod_{i=1}^{n} Q_{i}\left(s_{4,5}, \tau(d)\right) P_{i}\left(s_{2,5} s_{3,5}\right)=S
\end{aligned}
$$

Тогда (17) примет вид

$$
T(\tau(d))^{k} S=\psi_{2}^{k}\left(s_{4,5}, \tau(d)\right)
$$

Выполним сокращения в $T$ в свободной группе $\left\langle\tau(d), s_{2,5}, s_{3,5}, s_{4,5}\right\rangle$, причем сокращения между $Q_{i}$ и $\left(\tau(d) s_{4,5}^{-1}\right)^{t}$ будем вьполнять следующим образом. Если

$$
\left(Q_{i}\left(\tau(d) s_{4,5}^{-1}\right)^{t}\right)^{\eta}=\left(Q_{i}^{\prime} s_{4,5}^{-1}\left(\tau(d) s_{4,5}^{-1}\right)^{t^{\prime}}\right)^{\eta}, \quad\left|t^{\prime}\right|<|t|, \quad \eta= \pm 1
$$

либо

$$
\left(Q_{i}\left(\tau(d) s_{4,5}^{-1}\right)^{t}\right)^{\varepsilon}=\left(Q_{i}^{\prime}(\tau(d))^{-1}\left(\tau(d) s_{4,5}^{-1}\right)^{t^{\prime}}\right)^{\varepsilon}, \quad\left|t^{\prime}\right|<|t|, \quad \varepsilon= \pm 1,
$$

то в первом случае к $Q_{i}^{\prime}$ присоединяем $s_{4,5}^{-1}$, во втором $-(\tau(d))^{-1}$. При этом $\left|Q_{i}^{\prime}\right| \leqslant\left|Q_{i}\right|$. В результате вьполненных сокращений возможны следующие случаи:

1) $T=1$

2) $T=T_{1} P\left(s_{2,5}, s_{3,5}\right)$;

3) $T=T_{1} P\left(s_{2,5}, s_{3,5}\right)\left(\tau(d) s_{4,5}^{-1}\right)^{p} Q^{\prime}\left(\tau(d), s_{4,5}^{-1}\right)$;

4) $T=T_{1}\left(\tau(d) s_{4,5}^{-1}\right)^{p}, p \neq 0$.

В 1-м случае, так как $\sum_{j=1}^{n}\left|Q_{j}\right| \leqslant 2\left|F_{4}\right|<k$, для вьполнения равенства (17) в рассматриваемой свободной подгруппе необходимо, чтобы $P_{i}=1, i=1, \ldots, n$, так как в противном случае в левой части (17) будет стоять несократимое слово в свободных образуюших $s_{2,5}, s_{3,5}, s_{4,5}, \tau(d)$, а справа в $s_{4,5}, \tau(d)$.

Случай 2) аналогичен случаю 1$)$.

В случае 3 в в силу того, что $\left|Q^{\prime}\right| \leqslant 2\left|F_{4}\right|<k$, сокращение между $T$ и $(\tau(d))^{k}$ может дойти до $\left(\tau(d) s_{4,5}^{-1}\right)^{p}$ и сократится только один образующий $(\tau(d))^{\varepsilon}(\varepsilon= \pm 1)$, а между $(\tau(d))^{k}$ и $S$ полностью сократится $Q_{i}$. Но в силу выбора $k$ для вьполнения (17), как и в рассмотренных выше случаях, необходимо, чтобы $P_{i}=1, i=1, \ldots, n$.

Случай 4) аналогичен случаю 3). Из приведенных рассуждений следует доказательство леммы.

Лемма 11. Элемент $Z$, удовлетворяюший системе (5), имеет вид $Z=\Delta^{\prime 2 p}$ $\Delta^{2 n} L_{1}\left(\sigma_{1}^{4}, \sigma_{2}^{4}\right) L_{2}\left(\sigma_{4}^{2}, d\right)$, әде $\Delta^{\prime}-$ фундаментальный әлемент $B_{3}, \quad \Delta$ - фундаментальный әлемент $B_{5}, \quad p, n \in \mathbb{Z}$. 
ДокАЗАТЕльство. Имеем $Z=F_{1} F_{2} F_{3} F_{4}, F_{i} \in U_{i}$. По лемме $6 F_{1} F_{2}=\Delta^{\prime 2 k} \times$ $L_{1}\left(\sigma_{1}^{4}, \sigma_{2}^{4}\right)$, из леммы $7 \tau\left(F_{3}\right)=\left(s_{1,4} s_{2,4} s_{3,4}\right)^{t}$, а $F_{3}=\left(\sigma_{3} \sigma_{2} \sigma_{1}^{2} \sigma_{2} \sigma_{3}\right)^{t}$, где $t \in \mathbb{Z}$. Известно, что $\Delta_{B_{n+1}}^{2 b}=\left(\sigma_{n} \ldots \sigma_{3} \sigma_{2} \sigma_{1}^{2} \sigma_{2} \sigma_{3} \ldots \sigma_{n}\right)^{b} \Delta_{B_{n}}^{2 b}$. Тогда $\Delta^{2 t}=d^{t}\left(\sigma_{3} \sigma_{2} \sigma_{1}^{2} \sigma_{2} \sigma_{3}\right)^{t} \Delta^{2 t}$, откуда

$$
F_{3}=\Delta^{2 t} d^{-t} \Delta^{\prime-2 t}
$$

По лемме $10 F_{4}=L_{2}\left(\sigma_{4}^{2}, d\right)$. Поэтому

$$
Z=\Delta^{\prime 2 k} L_{1}\left(\sigma_{1}^{4}, \sigma_{2}^{4}\right) \Delta^{2 t} d^{-t} \Delta^{\prime-2 t} L_{2}\left(\sigma_{4}^{2}, d\right)=\Delta^{\prime 2 p} \Delta^{2 n} L_{1}\left(\sigma_{1}^{4}, \sigma_{2}^{4}\right) L_{2}\left(\sigma_{4}^{2}, d\right) .
$$

Из леммы 11 следует доказательство теоремы 3.

Пусть $H$ - произвольная конечно-порожденная подгруппа из $\Pi=\left\langle\sigma_{1}^{4}, \sigma_{2}^{4}\right\rangle \times\left\langle\sigma_{4}^{2}, d\right\rangle$ с образующими $a_{1}, a_{2}, \ldots, a_{n}$, имеющими вид

$$
a_{i}=L_{i}\left(\sigma_{1}^{4}, \sigma_{2}^{4}\right) M_{i}\left(\sigma_{4}^{2}, d\right), \quad i=1, \ldots, n .
$$

Подгруппу $H_{1}$, порожденную $L_{i}\left(\sigma_{1}^{4}, \sigma_{2}^{4}\right), i=1, \ldots, n$, будем назьвать проекцией $H$ на $\Pi_{1}=\left\langle\sigma_{1}^{4}, \sigma_{2}^{4}\right\rangle$, а подгруппу $H_{2}$, порожденную $M_{i}\left(\sigma_{4}^{2}, d\right), i=1, \ldots, n,-$ проекцией $H$ на $\Pi_{2}=\left\langle\sigma_{4}^{2}, d\right\rangle$.

ТЕОРЕМА 4. Если $H_{1}$ и $H_{2}$ не являются ииклическими, то $N_{R_{5}}(H) \subset N_{R_{5}}(\Pi)$.

ДокАЗАТЕЛЬСТво. Можно считать $L_{i}, i=1, \ldots, n$, свободными образующими. Пусть $Z \in N_{R_{5}}(H)$. Тогда

$$
Z^{-1} L_{i}\left(\sigma_{1}^{4}, \sigma_{2}^{4}\right) M_{i}\left(\sigma_{4}^{2}, d\right) Z=\varphi_{i}\left(\sigma_{1}^{4}, \sigma_{2}^{4}\right) \psi_{i}\left(\sigma_{4}^{2}, d\right), \quad i=1, \ldots, n .
$$

Представим $Z$ в виде $Z=F_{1} F_{2} F_{3} F_{4}$. Тогда (19) примет вид

$$
F_{4}^{-1} F_{3}^{-1} F_{2}^{-1} F_{1}^{-1} L_{i}\left(\sigma_{1}^{4}, \sigma_{2}^{4}\right) M_{i}\left(\sigma_{4}^{2}, d\right) F_{1} F_{2} F_{3} F_{4}=\varphi_{i}\left(\sigma_{1}^{4}, \sigma_{2}^{4}\right) \psi_{i}\left(\sigma_{4}^{2}, d\right), \quad i=1, \ldots, n .
$$

ЛЕмма 12. Система (20) әквивалентна системе

$$
\begin{gathered}
F_{2}^{-1} F_{1}^{-1} L_{i} F_{1} F_{2}=\varphi_{i}\left(\sigma_{1}^{4}, \sigma_{2}^{4}\right), \quad i=1, \ldots, n, \\
\left(F_{2}^{-1} F_{1}^{-1} L_{i}^{-1} F_{1} F_{2}\right) F_{3}^{-1}\left(F_{2}^{-1} F_{1}^{-1} L_{i} F_{1} F_{2}\right) F_{3}=1, \quad i=1, \ldots, n, \\
\left(F_{2}^{-1} F_{1}^{-1} L_{i}^{-1} F_{1} F_{2}\right) F_{4}^{-1}\left(F_{2}^{-1} F_{1}^{-1} L_{i} F_{1} F_{2}\right) \\
\times F_{3}^{-1} M_{i} F_{3} F_{4}=\psi_{i}\left(\sigma_{4}^{2}, d_{4}\right), \quad i=1, \ldots, n .
\end{gathered}
$$

ДоКАЗАТЕЛЬСТВо леммы аналогично доказательству леммы 4.

Аналогично доказательству лемм 6 и 7 доказьвается, что $F_{1} F_{2}=\Delta^{\prime 2 l} L_{1}\left(\sigma_{1}^{4}, \sigma_{2}^{4}\right)$, $l \in \mathbb{Z}, F_{3}=\left(\sigma_{3} \sigma_{2} \sigma_{1}^{2} \sigma_{2} \sigma_{3}\right)^{t}, t \in \mathbb{Z}, \tau\left(F_{3}\right)=\left(s_{1,4} s_{2,4} s_{3,4}\right)^{t}$.

Используя равенство (18), систему (23) можно переписать в виде

$$
\left(F_{2}^{-1} F_{1}^{-1} L_{i}^{-1} F_{1} F_{2}\right) F_{4}^{-1}\left(F_{2}^{-1} F_{1}^{-1} L_{i} F_{1} F_{2}\right) d^{t} M_{i} d^{-t} F_{4}=\psi_{i}\left(\sigma_{4}^{2}, d\right), \quad i=1, \ldots, n
$$

Обозначим $M_{i}^{\prime}=d^{t} M_{i} d^{-t}$. 
Лемма 13. Если $F_{4} \in U_{4}$ удовлетворяет системе $(24)$, то $F_{4}=F_{4}\left(\sigma_{4}^{2}, d\right)$.

ДокАЗАТЕЛЬСТво. Пусть снова $\left|F_{4}\right|$ - длина слова $F_{4} \in U_{4}$ в свободных образуюших $s_{2,5}, s_{3,5}, s_{4,5}, \tau(d)$. Возьмем $k \in \mathbb{N}$ такое, что $k>5\left|F_{4}\right|$. Возведем равенства $(19)$ в $k$-ю степень. Пусть $\tau\left(F_{4}\right)=\prod_{i=1}^{n} Q_{i}\left(s_{4,5}, \tau(d)\right) P_{i}\left(s_{2,5}, s_{3,5}\right)$, где $Q_{1}\left(s_{4,5}, \tau(d)\right)$ и $P_{n}\left(s_{2,5} s_{3,5}\right)$ могут быть равны единице.

Проводя рассуждения, аналогичные изложенньп в первой части доказательства леммы 10, и учитьвая лемму 12 , получаем равенства

$$
T_{i}\left(\tau\left(M_{i}^{\prime}\right)\right)^{k} S=\psi_{i}^{k}\left(\sigma_{4}^{2}, \tau(d)\right), \quad i=1, \ldots, n
$$

Выполнив в $T_{i}$ все указанные выше сокращения, получим следующие случаи.

1) $T_{i}=1, i \in\{1, \ldots, n\}$, т.е. $\left(\tau\left(M_{i}^{\prime}\right)\right)^{k} S=\psi_{i}^{k}\left(\sigma_{4}^{2}, \tau(d)\right), i \in\{1, \ldots, n\}$. Из этих равенств следует, что $S$ не содержит подслов вида $N\left(s_{2,5}, s_{3,5}\right)$.

2) $T_{i}=T_{1 i} P_{i}\left(s_{2,5}, s_{3,5}\right), i \in\{1, \ldots, n\}$. Подставляя в $(25)$, получаем, что $S$ не содержит подслов вида $N\left(s_{2,5}, s_{3,5}\right)$.

3) $T_{i}=T_{1 i} P_{i}\left(s_{2,5}, s_{3,5}\right) Q_{i}^{\prime}\left(s_{4,5}, \tau(d)\right), i \in\{1, \ldots, n\}$. В силу того, что $\sum\left|Q_{i}\right| \leqslant\left|F_{4}\right|$, для вьполнения (25) требуется, чтобы $S$ не содержало подслов вида $N\left(s_{2,5}, s_{3,5}\right)$.

В дальнейшем положим $R=\tau(d) s_{4,5}^{-1}$.

4) $T_{i}=T_{1 i} P_{i}\left(s_{2,5}, s_{3,5}\right) R^{p_{i}}$. В данном случае возможны два варианта:

a) $S$ не начинается на $Q\left(s_{4,5}, \tau(d)\right)$ для $i=1, \ldots, n$;

б) $S=Q\left(s_{4,5}, \tau(d)\right) P^{*}\left(s_{2,5}, s_{3,5}\right) S_{1}$.

Случай а) невозможен для всех $i$, так как все $M_{i}$ лежали бы в одной циклической группе, что противоречит условию.

Для случая б) равенство (25) имеет вид

$$
T_{1 i} P_{i}\left(s_{2,5}, s_{3,5}\right) R^{p_{i}}\left(\tau\left(M_{i}^{\prime}\right)\right)^{k} Q\left(s_{4,5}, \tau(d)\right) P^{*}\left(s_{2,5}, s_{3,5}\right) S_{1}=\psi_{i}^{k}\left(s_{4,5}, \tau(d)\right),
$$

где $S_{1}$ не начинается на $P^{*}\left(s_{2,5}, s_{3,5}\right)$. Для вьполнения данного равенства требуется, чтобы

$$
\left(\tau\left(M_{i}^{\prime}\right)\right)^{k}=R^{-p_{i}} Q^{-1}, \quad i \in\{1, \ldots, n\} .
$$

Из равенства (26) следует, что $\overline{M_{i}}=R^{\alpha_{i}}$ либо $\overline{M_{i}}=R^{* \alpha_{i}}$, где $R^{*}$ - циклическая перестановка $R, \overline{M_{i}}$ - циклически несократимая часть $M_{i}$, т.е. $M_{i}=A_{i} \overline{M_{i}} A_{i}^{-1}$. Пусть $\overline{M_{i}}=R^{\alpha_{i}}$ для всех $i$. По условию теоремы существует $M_{j}, j \neq i$, такое, что $M_{j}=B R^{\beta_{j}} B^{-1}, M_{i}=A R^{\alpha_{i}} A^{-1}, A \neq B R^{x}$, где $x \in \mathbb{Z}$. Выберем числа $\delta, \gamma \in \mathbb{Z}$ таким образом, чтобы произведение $M_{i}^{\delta} M_{j}^{\gamma}$ имело вид $a R^{n} b R^{m} c$, где $b$ отлично от единищы и не содержало ни слева, ни справа степени $R$. Очевидно, $Z=F_{1} F_{2} F_{3} F_{4}$ должно удовлетворять соотношению

$$
F_{4}^{-1} F_{3}^{-1} F_{2}^{-1} F_{1}^{-1}\left(L_{i}^{\delta} L_{j}^{\gamma} M_{i}^{\delta} M_{j}^{\gamma}\right)^{k} F_{1} F_{2} F_{3} F_{4}=\varphi_{i}^{\delta} \varphi_{j}^{\gamma} \psi_{i}^{\delta} \psi_{j}^{\gamma}
$$

Для равенства (27) проведем все вышеизложенные рассуждения. Тогда (25) примет вид

$$
T_{i j}\left(\tau\left(d^{t} M_{i}^{\delta} M_{j}^{\gamma} d^{-t}\right)\right)^{k} S=\psi_{i}^{\delta k} \psi_{j}^{\gamma k}, \quad t \in \mathbb{Z} .
$$


Но для этого равенства случай б) возможен только тогда, когда $S$ не содержит подслов $N\left(s_{2,5}, s_{3,5}\right)$, так как в данном случае сокращение не проходит через $\left(\tau\left(d^{t} M_{i}^{\delta} M_{j}^{\gamma} d^{-t}\right)\right)^{k}$.

Пусть теперь все $\overline{M_{i}}=R^{* \alpha_{i}}$. Тогда по условию теоремы вновь существует $M_{j}=$ $B R^{* \beta_{j}} B^{-1}, j \neq i$. Так как $M_{i}=A R^{* \alpha_{i}} A^{-1}$, можно выбрать числа $\delta, \gamma \in \mathbb{Z}$ таким образом, чтобы произведение $M_{i}^{\delta} M_{j}^{\gamma}$ имело вид $a R^{* n} b R^{* m} c$, где $b$ отлично от степени $R^{*}$, не равно единице и не содержит ни слева, ни справа степени $R^{*}$. В этом случае также можно показать, что соотношение вида (28) невозможно.

Пусть теперь $\overline{M_{i}}=R^{\alpha_{i}}, \overline{M_{j}}=R^{* \beta_{j}}$, т.е. $M_{i}=A R^{\alpha_{i}} A^{-1}$, a $M_{j}=B R^{* \beta_{j}} B^{-1}$. В данном случае также можно подобрать $\delta$ и $\gamma$ так, чтобы произведение $M_{i}^{\delta} M_{j}^{\gamma}$ имело вид $a R^{\alpha} b R^{* \beta} c$, так как по условию теоремы все $M_{i}$ не лежат в одной циклической группе. В этой ситуации для вьполнения (28) требуется, чтобы $S$ не содержало подслов $N\left(s_{2,5}, s_{3,5}\right)$.

5) Теперь рассмотрим случай, когда

$$
T_{i}=T_{1 i} P_{i}\left(s_{2,5}, s_{3,5}\right) R^{\alpha_{0 i}} Q_{1 i}^{\prime}\left(s_{4,5}, \tau(d)\right) R^{\alpha_{1 i}} \ldots Q_{n i}^{\prime}\left(s_{4,5}, \tau(d)\right) R^{\alpha_{n i}},
$$

где $\alpha_{n i}$ может быть равно нулю. Покажем, что в произведениях $Q_{j i}^{\prime} R^{\alpha i} Q_{(j+1) i}^{\prime}, 1 \leqslant$ $i<n$, и в $Q_{n i}^{\prime}\left(s_{4,5}, \tau(d)\right) R^{\alpha_{n i}}$ показатели $\alpha_{p i}$ не превосходят $\sum\left|Q_{i}\right|$. Действительно, в слове $\varphi^{-k}\left(s_{1,2}^{2}, s_{2,3}^{2}\right) P\left(s_{2,5}, s_{3,5}\right) \varphi^{k}\left(s_{1,2}^{2}, s_{2,3}^{2}\right)$ на основании равенств (1) $R$ возникает в суммарной нулевой степени, а поэтому при вьполнении сокращений $R$ с $Q_{i}\left(s_{4,5}, \tau(d)\right)$ в слове $T_{i}$ указанным вьше способом суммарная степень $R$ по модулю не превосходит $\sum\left|Q_{i}\right|$. Отсюда следует, что длина слова $Q_{1 i}^{\prime} R^{\alpha_{1 i}} \ldots Q_{n i}^{\prime} R^{\alpha_{n i}}$ ограничена длиной $2\left|F_{4}\right|$.

Если $\alpha_{0 i}$ равно $k$, то доказательство проводится аналогично случаю 4 . Если $\alpha_{0 i}$ мало, то доказательство очевидно. Лемма доказана.

ЛЕмма 14. Элемент $Z$, удовлетворяющий системе (19), имеет вид $Z=\Delta^{\prime 2 p} \times$ $\Delta^{2 n} L_{1}\left(\sigma_{1}^{4}, \sigma_{2}^{4}\right) L_{2}\left(\sigma_{4}^{2}, d\right)$.

ДокАЗАТЕЛЬСТво аналогично доказательству леммы 11.

Из леммы 14 следует доказательство теоремы.

Лемма 15 [6]. Пусть $H_{1}, H_{2}-$ две подгруппь из $B_{m}$. Если $H_{1}$ и $H_{2}$ сопряжсень в $B_{n}(n>m)$, то они сопряжены $и$ в $B_{m}$.

Пусть $a_{\nu}$ - образующие $H_{1}$. Из леммы 15 следует, что если $a_{\nu} z=z w_{\nu}\left(b_{\mu}\right)$, где $w_{\nu}\left(b_{\mu}\right)$ - слова из $H_{2}$, а $z \in B_{n}$, то существует элемент $z^{\prime} \in B_{m}$ такой, что

$$
a_{\nu} z^{\prime}=z^{\prime} w_{\nu}\left(b_{\mu}\right)
$$

Пусть $H-$ подгруппа из $B_{m}$ и $m<n$.

Лемма 16. Имеем $N_{B_{m}}(H) / \mathbb{C}_{B_{m}}(H) \simeq N_{B_{n}}(H) / \mathbb{C}_{B_{n}}(H)$.

ДоказАтЕльство. Пусть $r_{i}, i \in J,-$ представители смежных классов $N_{B_{m}}(H)$ по $\mathbb{C}_{B_{m}}(H)$, т.е. $N_{B_{m}}(H) / \mathbb{C}_{B_{m}}(H)=\bigcup_{i \in J} r_{i} \mathbb{C}_{B_{m}}(H)$. Зададим отображение $\varphi: N_{B_{m}}(H) / \mathbb{C}_{B_{m}}(H) \rightarrow N_{B_{n}}(H) / \mathbb{C}_{B_{n}}(H)$ следующим образом: $\varphi\left(r_{i} \mathbb{C}_{B_{m}}(H)\right)=$ $r_{i} \mathbb{C}_{B_{n}}(H)$. Проверим сюръективность данного отображения. Пусть $r_{0} \mathbb{C}_{B_{n}}(H) \in$ $N_{B_{n}}(H) / \mathbb{C}_{B_{n}}(H)$, где $r_{0} \in N_{B_{n}}(H)$. Если $a_{1}, \ldots, a_{n}, \ldots$ - образующие $H$, то

$$
r_{0} a_{i} r_{0}^{-1}=W_{i}\left(a_{\nu}\right) .
$$


По лемме 15 существует $r_{m}^{\prime} \in N_{B_{m}}(H)$ такое, что

$$
r_{m}^{\prime} a_{i} r_{m}^{\prime-1}=W_{i}\left(a_{\nu}\right)
$$

Из (29) и (30) имеем $r_{m}^{\prime-1} r_{0}=a_{i}^{-1} r_{m}^{\prime-1} r_{0} a_{i}$, откуда $a_{i} r_{m}^{\prime-1} r_{0}=r_{m}^{\prime-1} r_{0} a_{i}$, т.е. $r_{0}=$ $r_{m}^{\prime} z^{\prime}$, где $z^{\prime} \in \mathbb{C}_{B_{n}}(H)$. Пусть $r_{m}$ - представитель смежного класса, содержащего $r_{m}^{\prime}$. Тогда из последнего равенства $r_{0}=r_{m} z$, где $z \in \mathbb{C}_{B_{n}}(H)$. Таким образом, прообраз класса $r_{0} \mathbb{C}_{B_{n}}(H)$ есть класс $r_{m} \mathbb{C}_{B_{m}}(H)$. Инъективность данного отображения очевидна. Лемма доказана.

Теорема 5. Нормализатор подгруппь $H \subset B_{m}$ в $B_{n}, \quad n>m$, является расширением чентрализатора подгруппы $H$ в $B_{n}$ с помощью факторгруппь $N_{B_{m}}(H) / \mathbb{C}_{B_{m}}(H)$.

ДокАЗАТЕЛЬСтво следует из леммы 16. Из теоремы 5 получаем, что нормализаторы подгрупп, рассматриваемых в теоремах 3 и 4 , конечно порождены в группе $B_{n}$, $n>5$.

Выражаем благодарность рецензенту за внимательное отношение к работе.

\section{СПИСОК ЦИТИРОВАННОЙ ЛИТЕРАТУРЫ}

[1] Марков А. А. Основы алгебраической теории кос // Тр. МИАН СССР. 1945. Т. 16. C. $1-54$.

[2] Магнус В., Каррас А., Солитер Д. Комбинаторная теория групп. М.: Наука, 1974.

[3] Безверхний В. Н. Решение проблемы вхождения в некотором классе групп. I // Вопросы теории групп и гомологической алгебры. Ярославль, 1990. С. 40-52.

[4] Бардаков В. Г. К теории групп кос // Матем. сб. 1992. Т. 183. №6. С. 3-42.

[5] Гурзо Г. Г. О централизаторах конечных множеств элементов группы кос // Матем. заметки. 1985. Т. 37. № 1. С. 3-6.

[6] Безверхний В. Н., Добрынина И. В.О неразрешимости проблемы сопряженности подгрупп в группе крашеных кос $R_{5}$ // Матем. заметки. 1999. Т. 65. №1. С. 15-22.

[7] Михайлова К. А. Проблема вхождения для свободного произведения групп // Матем. сб. 1968. Т. 75. № 2. С. 199-210.

[8] Акименков А. М. О подгруппах группы кос $B_{4}$ // Матем. заметки. 1991. Т. 50. №6. C. $3-13$. 